\title{
Características pessoais e profissionais dos CEOS e a performance financeira: uma investigação nas empresas não financeiras listadas na B3
}

\section{Personal and professional characteristics of CEOS and financial performance: an investigation in non-financial companies listed in B3}

Recebimento: 26/10/2020 - Aceite: 08/01/2021 - Publicação: 01/02/2021 Processo de Avaliação: Double Blind Review

Inajá Allane Santos Garcia ${ }^{1}$ Doutoranda em Ciências Contábeis pela Universidade Federal da Paraíba (UFPB) Professora Assistente da Universidade Federal do Pará (UFPA) inajaallane@hotmail.com http://orcid.org/0000-0002-7844-9743

Juliane Simão de Andrade e Silva Graduada em Ciências Contábeis pela Universidade Federal da Paraíba (UFPB) julle_andrade@hotmail.com

\section{RESUMO}

O objetivo desse estudo foi analisar a influência das características pessoais e profissionais dos CEOs na performance financeira das empresas não financeiras de capital aberto listadas na B3. Para isso, adotou-se como suporte teórico a Teoria dos Escalões Superiores, proposta por Hambrick \& Mason (1984) e empiricamente utilizou-se um modelo empírico da regressão múltipla. Foram analisadas 328 empresas no período de 2011 a 2018, as quais formaram um painel desbalanceado com 824 observações. Para medir a performance financeira das empresas utilizou-se o retorno sobre o ativo (ROA) e as características pessoais e profissionais investigadas foram idade (IDA), gênero (GEN), experiência profissional (EXP), nível de escolaridade (ESC), área de formação (FOR) e tempo de mandato (TEM). As evidências apontam que CEOs femininas influenciam negativamente, enquanto CEOs com experiência anterior na Diretoria Executiva da mesma empresa e com maiores níveis de escolaridade influenciam positivamente no desempenho financeiro das

\footnotetext{
${ }^{1}$ Autor para correspondência: Universidade Federal da Paraíba (UFPB): Cidade Universitária - João Pessoa - PB - CEP: 58051-900- Brasil
} 
empresas de capital aberto da B3. Tais evidências contribuem com um público diverso, a exemplo das próprias empresas, CEOs que pretendem ingressar em empresas de capital aberto e ainda com os investidores e potenciais investidores.

Palavras-chave: características dos CEOs, B3, teoria dos escalões superiores.

\section{ABSTRACT}

The purpose of this study was to analyze the influence of CEOs' personal and professional characteristics on the financial performance of publicly traded non-financial companies listed on B3. For this, the Theory of Higher Levels, proposed by Hambrick and Mason (1984), was adapted as theoretical support and an empirical model of multiple regression was used. 328 companies were analyzed in the period from 2011 to 2018, which formed an unbalanced panel with 824 observations. To measure the financial performance of companies, return on assets (ROA) was used and the personal and professional characteristics investigated were age (IDA), gender (GEN), professional experience $(E X P)$, education level (ESC), area training (FOR) and tenure (TEM). Evidence indicates that female CEOs have a negative influence, while CEOs with previous experience in the Executive Board of the same company and with higher levels of education positively influence the financial performance of B3's publicly traded companies. Such evidence contributes to a diverse audience, such as the companies themselves, CEOs who intend to join publicly traded companies and also with investors and potential investors.

Keywords: Characteristics of CEOs, B3, Theory of Upper Echelons.

\section{INTRODUÇÃO}

A fim de tornar-se uma empresa competitiva no mercado e permanecer rentável, é necessário que haja comprometimento, habilidade, inteligência e dinâmica das pessoas que estão à frente da organização, sendo capazes de conduzir de forma satisfatória, ações estratégicas para atingir metas e atender aos objetivos organizacionais. Embora não quantificadas no balanço patrimonial de uma entidade, as características inerentes aos profissionais, em seus vários níveis hierárquicos, podem agregar valor à empresa. 
Essa perspectiva pode ser justificada pela Teoria dos Escalões Superiores (TES), desenvolvida por Hambrick \& Mason (1984), a qual preconiza que os resultados das entidades são vistos como reflexos de princípios e valores cognitivos de seus atores poderosos. Com base nessa teoria, os valores e princípios dos executivos do alto escalão, podem ser representados por suas características, as quais podem influenciar nas decisões estratégicas tomadas por esses profissionais e consequentemente a performance empresarial.

Dentre os executivos que compõe o Top Management Team (TMT), ou equipe do alto escalão, é possível destacar o Chief Executive Officer (CEO), que geralmente é visto como o ator mais importante e poderoso da organização (Minichilli, Corbetta, \& Macmillan, 2010), o qual está posicionado como líder da empresa e da equipe do alto escalão (Li \& Jones, 2018). Logo, acredita-se que as características desses executivos podem ter efeitos sob o desempenho financeiro das organizações via decisões estratégicas (Wang, Holmes, Oh, \& Zhu, 2016).

Desde o desenvolvimento da teoria, essa relação é o centro de análise de diversas pesquisas, a exemplo dos estudos internacionais de Henderson, Miller \& Hambrick (2006), Crossland \& Hambrick (2007), Minichilli et al. (2010), Guillet, Seo, Kucukusta \& Lee (2013), Peni (2014), Mohamed, Jarboui, Baccar \& Bouri (2015), Mio, Fasan \& Ros (2016), King, Srivastav \& Williams (2016), Kaur \& Singh (2018), Garcés-Galdeano \& GarcíaOlaverri (2019), Liu \& Jiang (2020), Naseem, Lin, Rehman, Ahmad \& Ali (2020) e nacionais como os de Serra, Três \& Ferreira (2016), Pacheco, Schmitt, Bortoluzzi, e Lunkes (2019) e Lunkes, Pereira, Santos \& Rosa (2019).

Porém, as evidências empíricas mostram resultados divergentes entre tais estudos. As evidências de Naseem et al. (2020) por exemplo, mostram que as características idade e gênero dos CEOs influenciam o desempenho das empresas, enquanto Mio et al. (2016) e Lunkes et al. (2019) relatam que tais características não são determinantes para o desempenho empresarial. Diante disso, a TES vem sendo investigada sob vários aspectos, os quais podem ser tomados como explicativos para tais contradições. De acordo com Abatecola \& Cristofaro (2020), a influência do CEO pode variar conforme o país no qual a empresa está localizada e é proporcional a discrição gerencial.

Com base nas reflexões apresentadas e considerando a ótica dos CEOs como atores capazes de influenciar o rumo empresarial, o presente trabalho busca responder a seguinte 
pergunta: qual a relação entre as características pessoais e profissionais dos CEOs e a performance financeira das empresas não financeiras de capital aberto listadas na Brasil Bolsa Balcão (B3)? A fim de responder tal questionamento, tem-se o objetivo de analisar a influência das características pessoais e profissionais dos CEOs na performance financeira das empresas não financeiras de capital aberto listadas na B3.

Tendo em vista a particularidade do mercado de capitais brasileiro, ainda considerado em desenvolvimento e com estrutura de propriedade concentrada nas mãos de poucos acionistas, optou-se por investigar as empresas que fazem parte do mesmo, com o intuito de encontrar evidências empíricas e verificar se mesmo diante dessa limitação, as características pessoais e profissionais dos CEOs podem influenciar no desempenho financeiro das organizações.

Ressalta-se que o presente estudo difere dos trabalhos nacionais encontrados sob mesma temática, como os de Serra et al. (2016), Lunkes et al. (2019) e Pacheco et al. (2019), pois além de considerar uma amostra de 328 empresas, diferentemente do tamanho da amostra desses autores, os quais foram 73, 104 e 29 empresas, respectivamente, considerou-se um período diferente de análise (2011 a 2018). Além disso, a importância deste artigo se dá devido a contribuição com a literatura a respeito da TES, mostrando a importância dos investidores considerarem também as características dos altos executivos ao avaliarem as organizações.

Nesse sentido, os resultados do presente estudo podem ser úteis às próprias firmas, as quais podem ser criteriosamente observadas e levadas em consideração quando no processo de contratação de novos CEOs que possuam o perfil adequando às estratégias pretendidas pelas empresas.

\section{REFERENCIAL TEÓRICO}

\subsection{O papel do CEO frente às decisões organizacionais e a Teoria dos Escalões}

\section{Superiores}

Os executivos do alto escalão possuem importância significativa no que se refere ao desempenho das empresas (Pacheco et al., 2019). No Brasil acredita-se que eles são um 
"fator-chave" para o desempenho de uma entidade (Serra et al., 2016). Porém, tais gestores possuem racionalidade limitada, objetivos variados e muitas vezes conflitantes, diferentes níveis de aspirações e formas de agir que são oriundas de suas interpretações, valores, crenças, conhecimentos que estes tomadores de decisão transportam para o aspecto administrativo (Finkelstein \& Hambrick, 1990).

Essa divergência de ações estratégicas pode ser explicada, pois, de acordo com Carpenter, Geletkanycz \& Sanders (2004), frente aos desafios para a tomada de decisão, a percepção dos altos gestores é construída através de seus valores e suas bases cognitivas. Todavia, à luz da TES, Hambrick \& Mason (1984) esclarece que devido a difícil observação, as características gerenciais observáveis podem servir como proxies e fornecer indicadores eficientes das construções psicológicas inobserváveis, podendo, portanto, representar tais valores e bases cognitivas.

No artigo seminal de Hambrick \& Mason (1984), os autores enfatizam a importância de entender as características dos altos executivos e como elas influenciam na tomada de decisão e, por consequência, nos resultados das entidades. Posteriormente, em uma revisitação a essa teoria, Hambrick (2007) ressaltou que a ideia central da TES possui duas partes interconectadas: a primeira considera que os executivos agem com base em suas interpretações personalizadas das situações estratégicas que enfrentam, e a segunda é que essas interpretações personalizadas são uma função das experiências, valores e personalidades dos executivos.

Conforme Carpenter et al. (2004), pesquisadores da área consideram o trabalho de Hambrick \& Mason (1984) um catalizador central. A partir de então, diversos estudos passaram a analisar o meio empresarial, explorando quais seriam e que tipo de variáveis poderiam influenciar os executivos a tomarem determinado rumo quanto às decisões estratégicas nas organizações e consequentemente nos resultados.

Percebe-se, portanto, que essa linha de pesquisa perdura desde o desenvolvimento da TES até os dias atuais, demonstrando o amadurecimento e consolidação da teoria nas pesquisas científicas. Mohamed et al. (2015), por exemplo, verificando os efeitos de características pessoais dos CEOs sobre o valor de empresas de transportes em 17 países no período de 2000 a 2011, encontraram evidências de que a idade e a formação técnica (educação em áreas não financeiras) podem influenciar positivamente o valor da empresa, e que CEOs com longos mandatos reduz o valor destas empresas. Contrariamente do que 
tais autores esperavam, a educação na área financeira não apresentou efeitos significativos no valor das empresas.

O estudo de Serra et al. (2016) verificou a influência das características sócio demográficas do CEO no desempenho das empresas brasileiras, no período de 1997 a 2012. Para isto, os autores utilizaram três indicadores financeiros (EBITDA, ROA e ROE) com base em 73 empresas e identificaram que as características idade, diversidade de experiência, experiência específica, longevidade no cargo e a competência do CEO (Nível de escolaridade) influenciam de forma diferente os indicadores de desempenho, e que o setor pode ser um fator significante para a relação das características dos CEOs com o desempenho das empresas. É importante salientar que esses autores não verificaram a influência das características gênero do CEO e dualidade de cargos dos CEOs, sendo essas as principais diferenças entre esse estudo e o presente artigo.

Em um trabalho mais atual, Pacheco et al. (2019) buscaram averiguar a existência ou não das relações entre as características demográficas dos executivos do alto escalão no desempenho de 29 empresas do setor de consumo não cíclico listadas na B3, no período de 2010 a 2016. Utilizando o ROA para avaliar o desempenho das entidades, os autores encontraram uma relação positiva entre o desempenho organizacional e as características gênero feminino e maior formação educacional, enquanto constataram que a idade e a familiaridade (percentual de parentesco do diretor com outro membro da diretoria ou do conselho de administração) estão relacionadas negativamente com o desempenho das empresas.

Visto alguns exemplos dos estudos de autores que tentaram verificar a importância das características dos CEOs, percebe-se que as mesmas têm efeitos significativos nas entidades, sejam positivas ou negativas. Isso ressalta a importância do estudo das características dos CEOs como delineadoras do desempenho empresarial.

\subsection{Características pessoais e profissionais dos CEOs e sua relação com a Performance Financeira}

Seguindo os estudos empíricos que abordaram a relação entre as características observáveis dos CEOs e o desempenho das organizações e com base na Teoria dos Escalões Superiores, a presente pesquisa analisa as seguintes características dos CEOs: idade, 
gênero, experiência profissional, nível de escolaridade, área de formação e tempo de mandato. O principal motivo para a escolha dessas variáveis foi a apresentação de justificativas da literatura para o relacionamento das mesmas com o desempenho das organizações e também o fato de tais informações estarem disponíveis no Formulário de Referência das empresas listadas na B3, os quais são divulgados pelas próprias empresas em seus sites oficiais e também no site da B3.

A idade de um executivo pode afetar a tendência de se envolverem em comportamentos de riscos, pois a medida que a idade avança, os CEOs tendem a se tornarem mais avessos ao risco e conservadores, diferentemente dos mais jovens (Naseem et al., 2020). Hambrick \& Mason (1984) sugerem que empresas que possuem gestores mais jovens possivelmente apresentarão um maior crescimento e variabilidade em sua lucratividade, por tenderem a tomar decisões mais arriscadas. Em contrapartida, os CEOs mais velhos podem ser mais experientes, e assim possuir mais vantagem competitiva quando comparado aos executivos mais jovens (Peni, 2014).

No que se refere ao gênero, de acordo com Peni (2014) a literatura psicológica acredita que as mulheres possuem uma melhor habilidade de se comunicar e tendem a superar os seus pares na solução de problemas em grupo e nas tomadas de decisão, além de serem mais conservadoras e avessas ao risco. Além disso, Mio et al. (2016) encontraram evidências em empresas da França, Alemanha e Reino Unido de que as CEOs femininos, geralmente possuem mais idades e experiências, o que pode indicar que estas precisam demonstrar maiores competências para chegar ao cargo. Logo, devido tais atribuições é de se esperar que elas superem os seus pares, porém os resultados de Mio et al. (2016) não demonstram efeitos significativos do gênero no desempenho financeiro, ao contrário dos resultados encontrados por Peni (2014), Pacheco et al. (2019) e Naseem et al. (2020), que afirmam que o gênero dos CEOs pode ser relevante.

Referente à experiência profissional, Finkelstein (1992) sugere que quanto maior for o número de posições que um gerente teve na entidade, maior será a sua interação com os fatores ambientais, proporcionando mais conhecimento para a tomada de decisão estratégica. Neste contexto, Wang et al. (2016) argumentam que a experiência do CEO em outras áreas, cargos ou funções iriam prepará-los para tomarem decisões estratégicas firmes, podendo os conceder mais conhecimento e confiança. 
Quanto à educação, Bai, Tsang \& Xia (2018) consideram que é um fator capaz de afetar a tomada de decisões gerenciais. Hambrick \& Mason (1984) apontam que se for reconhecido que grande parte das pessoas levam a sério suas decisões sobre educação, ela serve, até certo ponto, como um indicador dos seus valores e preferências cognitivas. Tais autores ainda sugerem que CEOs que possuem um maior nível de formação podem desempenhar melhor seus papéis. Garcés-Galdeano \& García-Olaverri (2019) concordam parcialmente, pois acreditam que tanto o nível, quanto a área são importantes, pois os CEOs que possuem um alto nível educacional tendem a ser mais proativos e autoconfiantes no que se refere ao processo de tomada de decisões arriscadas, podendo assim melhorar o desempenho inovador da entidade.

Quanto ao tempo de posse do CEO, Baysinger \& Hoskisson (1990) sugerem que o tempo de mandato de um executivo pode melhorar o desempenho financeiro de uma entidade, pois com o passar do tempo ele pode adquirir mais conhecimentos específicos, melhorando assim, sua capacidade de gerenciar. Além disso, tais executivos podem se beneficiar ao formar uma ampla rede de negócios durante seus longos mandatos (Peni, 2014).

Ressalta-se que tais características dos CEOs podem impactar de forma diferente o desempenho das empresas, logo, salienta-se a importância de estudar de forma mais ampla os efeitos dos CEOs na performance empresarial.

\section{METODOLOGIA}

\subsection{Definição da amostra e coleta dos dados}

A amostra inicial da presente pesquisa compreende todas as empresas não financeiras de capital aberto que compõe a Brasil, Bolsa, Balcão (B3), totalizando 328 empresas, as quais foram analisadas no período de 2011 a 2018. Ressalta-se que a análise das empresas que compõe a amostra foi feita considerando os anos de 2011 a 2018, tendo em vista a adoção da International Financial Reporting Standards (IFRS) em 2010 e a utilização de variáveis defasadas no cálculo dos indicadores de performance das firmas.

Os dados financeiros foram coletados por meio da base de dados da Economática ${ }^{\circledR}$ enquanto as variáveis referentes as características dos CEOs foram coletadas a partir da 
base de dados da Com Dinheiro e também do repositório de dados elaborado pelo Laboratório de Finanças e Risco (RiskFinLab) da FEA-USP (https://www.tatianaalbanez.com/basesexcel), cuja fonte primária são os Formulários de Referência disponibilizados no site da B3. É preciso destacar que as empresas do setor financeiro foram excluídas da amostra por possuírem uma estrutura de capital diferenciada das demais, o que poderia distorcer os resultados das análises ao inseri-las no modelo. Além das empresas financeiras foram excluídas também as observações que não apresentavam todos os dados para todas as variáveis em determinando ano, totalizando 824 observações em um conjunto de dados em painel desbalanceado.

\subsection{Modelo empírico e descrição das variáveis}

Tendo em vista que o objetivo desse estudo é analisar a influência das características pessoais e profissionais dos CEOs na performance financeira das empresas não financeiras de capital aberto listadas na B3, utilizou-se o modelo de regressão linear múltipla, por meio do método Ordinary Least Squares $(O L S)$, considerando a Equação 1:

$$
\begin{aligned}
R O A_{i, t}=\beta_{0}+ & \beta_{1} I D A_{i, t}+\beta_{2} G E N_{i, t}+\beta_{3} E X P_{i, t}+\beta_{4} E S C_{i, t}+\beta_{5} F O R_{i, t} \\
& +\beta_{6} \text { TEM }_{i, t}+\sum \beta_{n} \text { Controles }_{i, t}+\varepsilon_{i t}
\end{aligned}
$$

Em que:

$R O A_{i, t}=$ representa o retorno sobre o ativo da empresa i no ano $\mathrm{t}$;

$I D A_{i, t}=$ representa a idade do CEO da empresa i no ano $\mathrm{t}$;

$G E N_{i, t}=$ representa o gênero do CEO da empresa i no ano t;

$E X P_{i, t}=$ representa a experiência profissional do CEO da empresa i no ano t;

$E S C_{i, t}=$ representa o nível de escolaridade do CEO da empresa i no ano t;

$F O R_{i, t}=$ representa a área de formação do CEO da empresa i no ano t;

$T E M_{i, t}=$ representa o tempo de mandato do CEO da empresa i no ano t;

Controles $_{i, t}=$ representa o conjunto das variáveis de controle inseridas no modelo para a empresa i no ano t;

$\beta_{0}=$ representa a constante do modelo;

$\beta_{\mathrm{n}}=$ representam os parâmetros de cada uma das variáveis inseridas no modelo; 
$\varepsilon_{\mathrm{i}, \mathrm{t}}=$ representa $\mathrm{o}$ erro de estimação do modelo.

Tabela 1. Descrição das variáveis inseridas nas equações

\begin{tabular}{|c|c|c|c|}
\hline Variáveis & Operacionalização & $\begin{array}{c}\text { Sinal } \\
\text { Esperado }\end{array}$ & Base Teórica \\
\hline \multicolumn{4}{|c|}{ Dependente } \\
\hline $\begin{array}{l}\text { Retorno sobre o } \\
\text { Ativo (ROA) }\end{array}$ & $\begin{array}{l}\text { Lucro Líquido } \mathrm{i}_{\mathrm{i}, \mathrm{t}} / \text { Ativos } \\
\text { Totais } \mathrm{i}_{\mathrm{i}, \mathrm{t}-1}\end{array}$ & & $\begin{array}{l}\text { Crossland \& Hambrick (2007), Minichill } \\
\text { et al. } \text { (2010), Guillet } \text { et al. } \text { (2013), Peni } \\
\text { (2014) e Serra } \text { et al. (2016). }\end{array}$ \\
\hline \multicolumn{4}{|c|}{ Independentes } \\
\hline Idade (IDA) & Idade do CEO em anos & $(+)$ & $\begin{array}{l}\text { Peni (2014), Garcés-Galdeano \& García- } \\
\text { Olaverri (2019) e Liu \& Jiang (2020). }\end{array}$ \\
\hline Gênero (GEN) & $\begin{array}{l}\text { Variável dummy, sendo } 1 \text { caso } \\
\text { o CEO seja do sexo feminino, } \\
\text { e } 0 \text { caso contrário }\end{array}$ & $(+)$ & $\begin{array}{l}\text { Peni (2014), Mio et al. (2016), Kaur \& } \\
\text { Singh (2018) e Naseem et al. (2020). }\end{array}$ \\
\hline $\begin{array}{l}\text { Experiência } \\
\text { profissional (EXP) }\end{array}$ & $\begin{array}{c}\text { Variável dummy, sendo } 1 \text { caso } \\
\text { o CEO tenha trabalhado na } \\
\text { mesma empresa em outros } \\
\text { cargos da Diretoria Executiva e } \\
\text { 0 caso contrário }\end{array}$ & $(+)$ & Serra et al. (2016) e Lunkes et al. (2019). \\
\hline $\begin{array}{l}\text { Nível de escolaridade } \\
\text { (ESC) }\end{array}$ & $\begin{array}{l}\text { Nível mais alto de escolaridade } \\
\text { do CEO, sendo } 0 \text { quando o } \\
\text { CEO possui nível de } \\
\text { escolaridade abaixo da } \\
\text { graduação, } 1 \text { quando o CEO } \\
\text { possui graduação, } 2 \text { quando } \\
\text { possui pós graduação lato } \\
\text { sensu, } 3 \text { quando possui } \\
\text { Mestrado e } 4 \text { quando possui } \\
\text { Doutorado }\end{array}$ & $(+)$ & $\begin{array}{l}\text { King et al. (2016), Kaur \& Singh (2018), } \\
\text { adaptado de Garcés-Galdeano \& García- } \\
\text { Olaverri (2019). }\end{array}$ \\
\hline $\begin{array}{l}\text { Área de formação do } \\
\text { CEO (FOR) }\end{array}$ & $\begin{array}{l}\text { Variável dummy, sendo } 1 \text { caso } \\
\text { o CEO tenha formação na área } \\
\text { de Administração, Economia } \\
\text { ou Ciências Contábeis, e } 0 \\
\text { caso contrário }\end{array}$ & $(+)$ & $\begin{array}{l}\text { Mio et al. (2016), Sprenger, Kronbauer e } \\
\text { Costa (2017), Garcés-Galdeano \& García- } \\
\text { Olaverri (2019) e Naseem et al. (2020). }\end{array}$ \\
\hline $\begin{array}{l}\text { Tempo de Mandado } \\
\text { (TEM) }\end{array}$ & $\begin{array}{l}\text { Número de anos de posse no } \\
\text { cargo de CEO da empresa }\end{array}$ & $(+)$ & $\begin{array}{l}\text { Peni (2014), Garcés-Galdeano \& García- } \\
\text { Olaverri (2019), Liu \& Jiang (2020) e } \\
\text { Naseen } \text { et al. (2020). }\end{array}$ \\
\hline \multicolumn{4}{|c|}{ Controles } \\
\hline $\begin{array}{l}\text { Tamanho da empresa } \\
\text { (TAM) }\end{array}$ & $\begin{array}{l}\text { Ln do Ativo total das empresas } \\
\text { no ano t }\end{array}$ & $(+)$ & $\begin{array}{l}\text { Finkelstein \& Hambrick (1990), } \\
\text { Henderson } \text { et al. (2006), Peni (2014) e Liu } \\
\text { \& Jiang (2020). }\end{array}$ \\
\hline $\begin{array}{l}\text { Dualidade de cargos } \\
\text { (DUA) }\end{array}$ & $\begin{array}{l}\text { Variável Dummy, sendo } 1 \text { caso } \\
\text { o CEO ocupa cargos de CEO e } \\
\text { presidente ao mesmo tempo, e } \\
0 \text { caso contrário }\end{array}$ & $(+/-)$ & $\begin{array}{l}\text { Guillet et al. (2013), Peni (2014), Pham, } \\
\text { Oh \& Pech (2015), Bai et al. (2018), } \\
\text { Kaur \& Singh (2019) e Naseem } \text { et al. } \\
\text { (2020). }\end{array}$ \\
\hline
\end{tabular}

Note. Fonte: Elaborado pelas autoras (2020). 
Para melhor esclarecimento acerca das variáveis utilizadas nas equações, elaborouse a Tabela 1, a qual contempla cada uma delas, bem como sua forma de operacionalização e as respectivas bases teóricas que utilizaram tais variáveis em estudos anteriores.

Tais variáveis apresentadas na Tabela 1 são utilizadas para compor as equações estimadas, bem como apresentam os sinais esperados para cada uma das relações entre as variáveis independentes e de controle.

\subsection{Variável dependente e independentes}

O ROA é uma variável contábil que mede a capacidade de retorno obtida mediante a utilização dos ativos da entidade e frequentemente é utilizada em estudos que tentam verificar a influência das características dos CEOs no desempenho financeiro das empresas, como por exemplo, nos estudos de Crossland \& Hambrick (2007), Minichilli et al. (2010), Peni (2014), Serra et al. (2016) e Kaur \& Singh (2018). Com base nessas variáveis, o presente estudo calculou e analisou o desempenho financeiro das 328 empresas que compõe a amostra do estudo.

As variáveis independentes que são de interesse desse estudo foram aquelas que representam as características pessoais e profissionais dos CEOs, explicadas na Tabela 1, como: idade, gênero, experiência profissional, nível de escolaridade, área de formação e tempo de mandato.

No que se refere à idade (IDA) do CEO, espera-se que executivos mais velhos influenciem positivamente o resultado financeiro das empresas, apoiando-se nos estudos de Wang et al. (2016) e Pacheco et al. (2019) que perceberam que os CEOs mais velhos estão associados a melhores resultados de desempenho das firmas, logo, espera-se uma relação positiva.

Quanto ao gênero (GEN) do CEO, sugere-se que o feminino esteja relacionado positivamente ao resultado financeiro das empresas, assim como encontrado nos trabalhos de Peni (2014) e Pacheco et al. (2019), pois baseando-se na literatura acredita-se que sejam mais conservadoras, possuam maiores habilidades de comunicação e de trabalhos em grupos, além de geralmente serem avessas ao risco.

A experiência profissional (EXP) do CEO representa a diversidade de cargos anteriores ocupados por um CEO dentro de uma mesma organização, que os proporciona mais conhecimento, e consequentemente afete positivamente o desempenho empresarial. 
Esta sugestão se baseia na ideia de Finkelstein (1992), o qual supõe que diferentes cargos ocupados por um CEO, conforme progridem em sua carreira, podem fornecer dados importantes tanto da empresa quanto do seu ambiente, e na de Lunkes et al. (2019), que sugerem que gestores promovidos internamente possuem uma melhor capacidade para desenvolver e implementar ações que respondam as demandas de gerentes dos outros níveis.

Quanto ao nível de escolaridade (ESC) do CEO, espera-se que quanto maior for, melhor será o desempenho da empresa por ele controlada, ou seja, uma relação positiva. Seguindo os achados de King et al. (2016), os quais obtiveram evidências de que o nível educacional de um CEO é importante para o desempenho da empresa.

Com relação a área de formação (FOR) do CEO, espera-se uma relação positiva entre os CEOs que possuem uma formação da área de negócios e o desempenho financeiro da empresa. Nesse sentido, Garcés-Galdeano \& García-Olaverri (2019) constataram relevância quanto ao tipo de formação, e afirmaram que CEOs com uma educação na área de negócios, estão associados a bons desempenhos, pois tais conhecimentos podem atribuir uma melhor visão e influência, auxiliando assim a tomarem decisões de forma eficiente.

Espera-se que CEOs com mais tempo de mandato (TEM) estejam associados positivamente a melhores resultados, pois enseja que possuam mais experiências e conhecimentos específicos do setor, e assim possam responder de forma mais eficiente às situações empresariais. Os resultados obtidos por Peni (2014) confirmam este argumento, pois a autora encontrou uma relação positiva e estatisticamente significante entre o tempo de mandato dos executivos e o desempenho financeiro das empresas.

\section{ANÁLISE E DISCUSSÕES}

Antes da estimação dos modelos empíricos, representado em sua forma completa pela Equação 1, seguindo o método OLS, foi realizado o teste Variance Inflation Factor para verificar se existia multicolinearidade entre as variáveis presentes, o qual não apresentou maiores problemas, sendo que o maior VIF foi 1,17 referente a variável Idade do CEO. Para a estimação da regressão foram realizados os testes de Chow, Hausman e Breusch-Pagan, os quais apontaram que o modelo de painel que melhor se adequa aos dados é o Pooled Ordinary Least Squares (POLS). Além desses, foram realizados também os testes para verificação de autocorrelação (teste de Wooldridge), heterocedasticidade 
(teste de Breusch-Pagan/Cook-Weisberg), normalidade dos resíduos (teste de JarqueBera) e multicolinearidade (Variance Inflation Factor).

No que se refere aos resultados das estimações dos modelos empíricos, optou-se por estimá-los utilizando duas equações, uma que contempla apenas as variáveis de interesse deste estudo e outra com a inserção das variáveis de controle, conforme evidenciado no item 3.2 desse estudo. De tal forma, os resultados oriundos dessas estimações podem ser analisados a partir dos dados da Tabela 2.

Tabela 2. Estatísticas inferenciais do modelo, referente ao período de 2011 a 2018

\begin{tabular}{|c|c|c|c|c|c|c|}
\hline \multirow{2}{*}{ Variáveis } & \multicolumn{3}{|c|}{$\begin{array}{c}\text { Equação (1) } \\
824 \text { Observações }\end{array}$} & \multicolumn{3}{|c|}{$\begin{array}{c}\text { Equação (2) } \\
824 \text { Observações }\end{array}$} \\
\hline & Coef. & $\begin{array}{c}\text { Erro } \\
\text { Padrão }\end{array}$ & p-valor & Coef. & Erro Padrão & p-valor \\
\hline CONST. & $-0,786$ & 0,528 & 0,137 & $-1,114$ & 0,196 & 0,000 \\
\hline IDA & $-0,001$ & 0,008 & 0,894 & $-0,003$ & 0,002 & 0,262 \\
\hline GEN & $-2,207$ & 0,478 & 0,000 & $-0,397$ & $\mathbf{0 , 1 3 0}$ & 0,002 \\
\hline EXP & 0,463 & 0,180 & 0,010 & 0,095 & 0,048 & 0,048 \\
\hline ESC & $\mathbf{0 , 2 3 9}$ & 0,113 & $\mathbf{0 , 0 3 5}$ & $-0,001$ & 0,031 & 0,968 \\
\hline FOR & 0,104 & 0,190 & 0,586 & 0,042 & 0,051 & 0,405 \\
\hline TEM & 0,017 & 0,017 & 0,309 & 0,006 & 0,004 & 0,159 \\
\hline TAM & & & & 0,076 & 0,010 & 0,000 \\
\hline \multirow[t]{2}{*}{ DUA } & & & & 0,028 & 0,059 & 0,631 \\
\hline & \multicolumn{3}{|c|}{$\mathrm{R}^{2}=0,035$} & \multicolumn{3}{|c|}{$\mathrm{R}^{2}=0,089$} \\
\hline
\end{tabular}

Note. Fonte: Elaborado pelas autoras (2020).

Por meio da Equação (1) verifica-se que as variáveis gênero (GEN), experiência profissional (EXP) e nível de escolaridade (ESC) foram significantes com relação ao desempenho financeiro das empresas de capital aberto da B3 no período de 2011 a 2018.

No que se refere a variável de gênero, a relação encontrada foi significativa e contrária ao que era esperado, remetendo a uma diminuição do desempenho da empresa quando o cargo de CEO é ocupado por pessoas do gênero feminino. Tal relação é expressiva, tendo em vista que o coeficiente foi de $-2,207$ e o nível de significância foi de $1 \%$. 
De acordo com as evidências empíricas, há uma significância estatística positiva de $5 \%$ entre a experiência profissional do gestor e o desempenho financeiro, conforme era esperado. Isso significa que o fato do CEO já ter trabalhado na empresa ocupando outros cargos na Diretoria Executiva antes de ser CEO, ajuda a aumentar o desempenho da empresa, o que pode ser explicado devido ao melhor conhecimento sobre como funcionam os processos e dinâmicas da empresa. Nessa perspectiva estão os argumentos de Lunkes et al. (2019), os quais apontam que gestores promovidos internamente melhoram suas capacidades para desenvolver e implementar ações que respondam a diversas demandas.

O nível de escolaridade está positivamente relacionado com o desempenho das empresas de capital aberto no mercado brasileiro, isso significa que quanto maior o nível de escolaridade dos CEOs, maior tende a ser o resultado das organizações. Os estudos de King et al. (2016) e Garcés-Galdeano \& García-Olaverri (2019) também encontraram resultados semelhantes, em que o nível de escolaridade afeta positivamente o resultado da empresa. Tal evidência pode ser justificada porque espera-se que pessoas com maior escolaridade possuam mais e melhores conhecimentos, pois isso os tornariam mais capacitados e com maiores condições de execução das suas funções. Nesse sentido, GarcésGaldeano \& García-Olaverri (2019) complementam que CEOs com nível educacional elevado são mais autoconfiantes e proativos com relação as suas ações e isso pode contribuir para o aumento dos resultados das empresas.

Quando se verificam os resultados na Equação (2), percebe-se que apenas as relações das variáveis gênero do CEO e experiência se mantiveram, dando indício de não consistência dos resultados quanto a relação dessa variável com o desempenho das empresas ao inserir outros fatores. Das duas variáveis de controle que foram inseridas, apenas o tamanho da empresa foi significante em $1 \%$, inferindo que quanto maior a empresa melhor tende a ser o desempenho financeiro das empresas de capital aberto listadas na B3.

Ademais, as variáveis idade do CEO, formação e tempo de mandato, que também representam características pessoais e profissionais dos CEOs, se mostraram insignificantes, ou seja, não possuem capacidade de influenciar no desempenho financeiro das empresas. Tal resultado pode ser justificado devido a concentração de propriedade que as empresas do mercado brasileiro possuem, pois isso pode fazer com que a discrição 
gerencial diminua e consequentemente diminua a influência que tais CEOs possuem sobre os resultados das companhias.

\section{CONSIDERAÇÕES FINAIS}

A presente pesquisa tinha o intuito de responder ao seguinte problema de pesquisa: Qual a relação entre as características pessoais e profissionais dos CEOs e a performance financeira das empresas não financeiras de capital aberto listadas na B3?

Foram analisadas algumas características pessoais e profissionais, como idade, gênero, experiência profissional, nível de escolaridade, área de formação e tempo de mandato, amparadas teoricamente pela Teoria dos Escalões Superiores, de Hambrick \& Mason (1984). Por meio da análise dos dados referentes a 328 empresas listadas na B3, pode-se observar que algumas dessas características pessoais e profissionais dos gestores têm o poder de influenciar na performance financeira das empresas, como por exemplo o gênero, a experiência profissional e o nível de escolaridade. As evidências apontam que CEOs do gênero feminino tendem a influenciar de maneira negativa o desempenho financeiro das organizações. Por outro lado, a performance financeira é influenciada positivamente por CEOs que já tiveram outros cargos na Diretoria Executiva da mesma empresa que são CEOs e também por CEOs com maiores níveis de escolaridade.

Percebe-se portanto, que nem todas as características pessoais e profissionais dos CEOs conseguem impactar de alguma forma o desempenho das empresas, remetendo a questionamentos à própria TES, quanto a sua validade. Porém, já era previsto pelo próprio Hambrick (2007) a respeito disso, ao argumentar que a discrição gerencial e as demandas de cargos executivos, as quais se diferenciam de empresa para empresa, podem interferir no impacto que tais características causam no desempenho das firmas.

Mesmo assim, cabe aqui ressaltar as contribuições do presente estudo, o qual pode auxiliar as empresas que pretendem contratar novos CEOs, ajudando-as a montar um perfil de CEO que esteja mais alinhado as suas necessidades e competências. Isso porque o presente artigo apresenta que algumas características podem influenciar diretamente nos resultados das companhias. Além disso, esse estudo contribui com CEOs que pretendem ingressar em companhias de capital aberto, dando indícios de características que atraem as 
empresas, como a experiência profissional e o nível de escolaridade por exemplo. Sob outra perspectiva, os resultados desse estudo auxiliam os investidores e potenciais investidores, tendo em vista que revela que algumas características dos gestores influenciam o desempenho financeiro das empresas e consequentemente influenciam o retorno dos seus investimentos. Logo, tais resultados servem aos investidores para que os mesmos consigam fazer melhores previsões dos seus retornos futuros ao analisarem as características dos CEOs.

Por fim, sugere-se que a continuidade desse estudo seja feita no sentido de identificar outras características ou até mesmo outros fatores que podem estar bloqueando a influência dos CEOs na tomada de decisões estratégicas e consequentemente no desempenho financeiro das firmas.

\section{REFERÊNCIAS}

Abatecola, G., \& Cristofaro, M. (2020). Hambrick and Mason's "upper echelons theory": evolution and open avenues. Journal of Management History, 26(1), 116-136. https://doi.org/10.1108/JMH-02-2018-0016.

Bai, X., Tsang, E. W. K., \& Xia, W. (2018). Domestic versus foreign listing: Does a CEO's educational experience matter? Journal of Business Venturing, 35(1), 1-30. https://doi.org/10.1016/j.jbusvent.2018.10.004.

Baysinger, B., \& Hoskisson, R. E. (1990). The composition of boards of directors and strategic control: Effects on corporate strategy. The Academy of Management Review, 15(1), 72-87. https://doi.org/10.2307/258106.

Carpenter, M. A., Geletkanycz, M. A., \& Sanders, W. G. (2004). Upper echelons research revisited: antecedents, elements, and consequences of top management team composition. Journal of Management, 30(6), 749-778. https://doi.org/10.1016/j.jm.2004.06.001.

Crossland, C., \& Hambrick, D. C. (2007). How national systems differ in their constraints on corporate executives: a study of CEO effects in three countries. Strategic Management Journal, 28(8), 767-789. https://doi.org/10.1002/smj.610.

Finkelstein, S. (1992). Power in top management teams: dimensions, measurement, and validation. The Academy of Management Journal, 35(3), 505-538. https://doi.org/10.2307/256485. 
Finkelstein, S., \& Hambrick, D. C. (1990). Top Management Team tenure and organizational outcomes: the moderating role of managerial discretion. Administrative Science Quarterly, 35(3), 484-503. https://doi.org/10.2307/2393314.

Garcés-Galdeano, L., \& García-Olaverri, C. (2019). The hidden value of intangibles: do CEO characteristics matter? International Journal of Manpower, 40(6), 1075-1091. https://doi.org/10.1108/IJM-06-2018-0199

Guillet, B. D., Seo, K., Kucukusta, D., \& Lee, S. (2013). CEO duality and firm performance in the U.S. restaurant industry: Moderating role of restaurant type. International Journal of Hospitality Management, 33(1), 339-346. https://doi.org/10.1016/j.ijhm.2012.10.004

Hambrick, D. C., \& Mason, P. A. (1984). Upper echelons: the organization as a reflection of its top managers. The Academy of Management Review, 9(2), 193-206. https://doi.org/10.2307/258434

Hambrick, D. C. (2007). Upper echelons theory: An update. Academy of Management Review, 32(2), 334-343. https://doi.org/10.2307/20159303

Henderson, A. D., Miller, D., \& Hambrick, D. C. (2006). How quickly do CEOs become obsolete? Industry Dynamism, CEO Tenure, and Company Performance. Strategic Management Journal, 27(5), 447-460. https://doi.org/10.1002/smj.524

Kaur, R., \& Singh, B. (2018). CEOs' Characteristics and Firm Performance: A Study of Indian Firms. Indian Journal of Corporate Governance, 11(2), 1-16. https://doi.org/10.1177/0974686218806714

King, T., Srivastav, A., \& Williams, J. (2016). What's in an education? Implications of CEO education for bank performance. Journal of Corporate Finance, 37, 287-308. https://doi.org/10.1016/j.jcorpfin.2016.01.003

Li, M., \& Jones, C. D. (2018). The effects of TMT faultlines and CEO-TMT power disparity on competitive behavior and firm performance. Group \& Organization Management, 44(5), 874-914. https://doi.org/10.1177/1059601118813790

Liu, C., \& Jiang, H. (2020). Impact of CEO characteristics on firm performance: evidence from China listed firms. Applied Economics Letters, 27(14), 1-5. https://doi.org/10.1080/13504851.2019.1607965

Lunkes, R. J., Pereira, B. D. S., Santos, E. A., \& Rosa, F. S. (2019). Analysis of the relationship between the observable characteristics of CEOs and organizational performance. Contaduría y administración, $\quad 64(4), \quad 1-21$. https://doi.org/10.22201/fca.24488410e.2018.1785

Minichilli, A., Corbetta, G., \& Macmillan, I. C. (2010). Top management teams in familycontrolled companies: 'familiness','faultlines', and their impact on financial performance. 
Journal of Management Studies, 47(2), 205-222. https://doi.org/10.1111/j.14676486.2009.00888.x

Mio, C., Fasan, M., \& Ros, A. (2016). Owners' preferences for CEOs characteristics: did the world change after the global financial crisis? Corporate Governance: The International Journal of Business in Society, 16(1), 116-134. https://doi.org/10.1108/CG07-2015-0092

Mohamed, E. B., Jarboui, S., Baccar, A., \& Bouri, A. (2015). On the effect of CEOs' personal characteristics in transport firm value? A stochastic frontier model. Case Studies on Transport Policy, 3(2), 176-181. https://doi.org/10.1016/j.cstp.2015.01.001

Naseem, M. A., Lin, J., Rehman, R., Ahmad, M. I., \& Ali, R. (2020). Does capital structure mediate the link between CEO characteristics and firm performance? Management Decision, 58(1), 164-181. https://doi.org/10.1108/MD-05-2018-0594

Pacheco, J., Schmitt, M., Bortoluzzi, D. A., \& Lunkes, R. J. (2019). Características dos executivos do alto escalão e a influência no desempenho: Um estudo em empresas listadas na Bolsa Brasileira. BASE - Revista de Administração e Contabilidade Da Unisinos, 16(1), 59-83. https://doi.org/10.4013/base.2019.161.03

Peni, E. (2014). CEO and chairperson characteristics and firm performance. Journal of Management \& Governance, 18(1), 185-205. https://doi.org/10.1007/s10997-012-9224-7

Pham, N., Oh, K. B., \& Pech, R. (2015). Mergers and acquisitions: CEO duality, operating performance and stock returns in Vietnam. Pacific-Basin Finance Journal, 35(A), 298316. https://doi.org/10.1016/j.pacfin.2015.01.007

Serra, F. R., Três, G., \& Ferreira, M. P. (2016). The "CEO" Effect on the performance of Brazilian companies: An empirical study using measurable characteristics. European Management Review, 13(3), 193-205. https://doi.org/10.1111/emre.12079

Sprenger, K. B., Kronbauer, C. A., \& Costa, C. M. (2017). Características do CEO e o Gerenciamento de Resultados em empresas listadas na BM\&FBOVESPA. Revista Universo Contábil, 13(3), 120-142. https://doi.org/10.4270/ruc.2017321

Wang, G., Holmes, R. M., Jr., Oh, I. -S., \& Zhu, W. (2016). Do CEOs matter to firm strategic actions and firm performance? A meta-analytic investigation based on upper $\begin{array}{llll}\text { echelons theory. Personnel } & \text { 775-862. }\end{array}$ https://doi.org/10.1111/peps.12140 